\title{
Infection Risk in Patients with Multiple Myeloma during the Era of Monoclonal Antibody Therapy
}

Stephanie J. Hoffman, M.D. ${ }^{1}$, Malathi Kandarpa, Ph.D. ${ }^{2}$, Jing Christine Ye, M.D. ${ }^{2}$

${ }^{1}$ Michigan Medicine, Department of Internal Medicine, Ann Arbor, MI, USA

2Michigan Medicine Rogel Cancer Center, Department of Internal Medicine, Division of Hematology/Oncology, Ann Arbor, MI, USA

\section{CONTEXT}

With the advent of new monoclonal antibody therapies for Multiple Myeloma (MM), the impact of their mechanisms of immunosuppression on the risk and types of infections developed by patients who receive them are not well understood. While initial randomized control trial data suggests increased rates of Varicella Zoster Virus (elotuzumab, daratumumab) and lymphopenia (elotuzumab), more data is necessary to delineate whether practice changes in routine prophylaxis are indicated.

\section{OBJECTIVE}

To identify the incidence and type of infection in patients with MM receiving elotuzumab or daratumumab-based regimens, describe their characteristics, and compare these data points to patients with MM who did not receive regimen to help inform decisionmaking regarding appropriate infection prophylaxis.

\section{METHODS}

This was a retrospective chart review including patients treated from January 1, 2002 - April 30, 2018 at an academic referral center. Patients with MM who were 18 years or older and who received therapy incorporating daratumumab, elotuzumab, or both were eligible for inclusion in the "intervention" arm. The "control" group was comprised of patients with MM in the same age group who received regimens that did not include monoclonal antibody therapy.
DATADIRECT database query using these parameters was performed and resulted in the identification of 58 patients for the intervention group and 56 for the control group, of whom 51 and 44 met criteria for inclusion, respectively.

\section{Table 1. Baseline characteristics}

Interventions were not standardized and patients received a range of combination therapy in the relapsed/refractory setting. The primary outcome measures were formulated prior to data collection: presence/type of infection, time to infection from initiation of monoclonal antibody based therapy in the intervention group versus initiation of treatment in the control group. Secondary outcome measures were infection-associated mortality and use of relevant prophylaxis.

\section{RESULTS}

Baseline characteristics are summarized in Table 1. Of the 51 patients in the intervention group, 15 received elotuzumab and daratumumab consecutively. Of those who received elotuzumab, $16.7 \%$ versus $16.3 \%$ of those who received daratumumab experienced infection.
Overall, $21.6 \%$ of patients developed infections including 5 with bacteria pneumonia, one case of JC virus (only episode associated with mortality) and remaining with neutropenic fever diverticulitis, or diarrhea. All patients received anti-viral prophylaxis. The mean time to infection was $3.5 \pm 5.5$ months.

\section{CONCLUSIONS}

The frequency of infections was relatively low in the intervention group with the majority being bacterial pneumonias (only 1 of 5 in the setting of neutropenia), whereas infections occurred in over half of control patients (although several in the post-transplant setting). Further studies with increased sample size are needed to provide definitive answers regarding the selection of prophylactic medications with these monoclonal antibody therapies.

Antibody-dependen cellular cytotoxicity
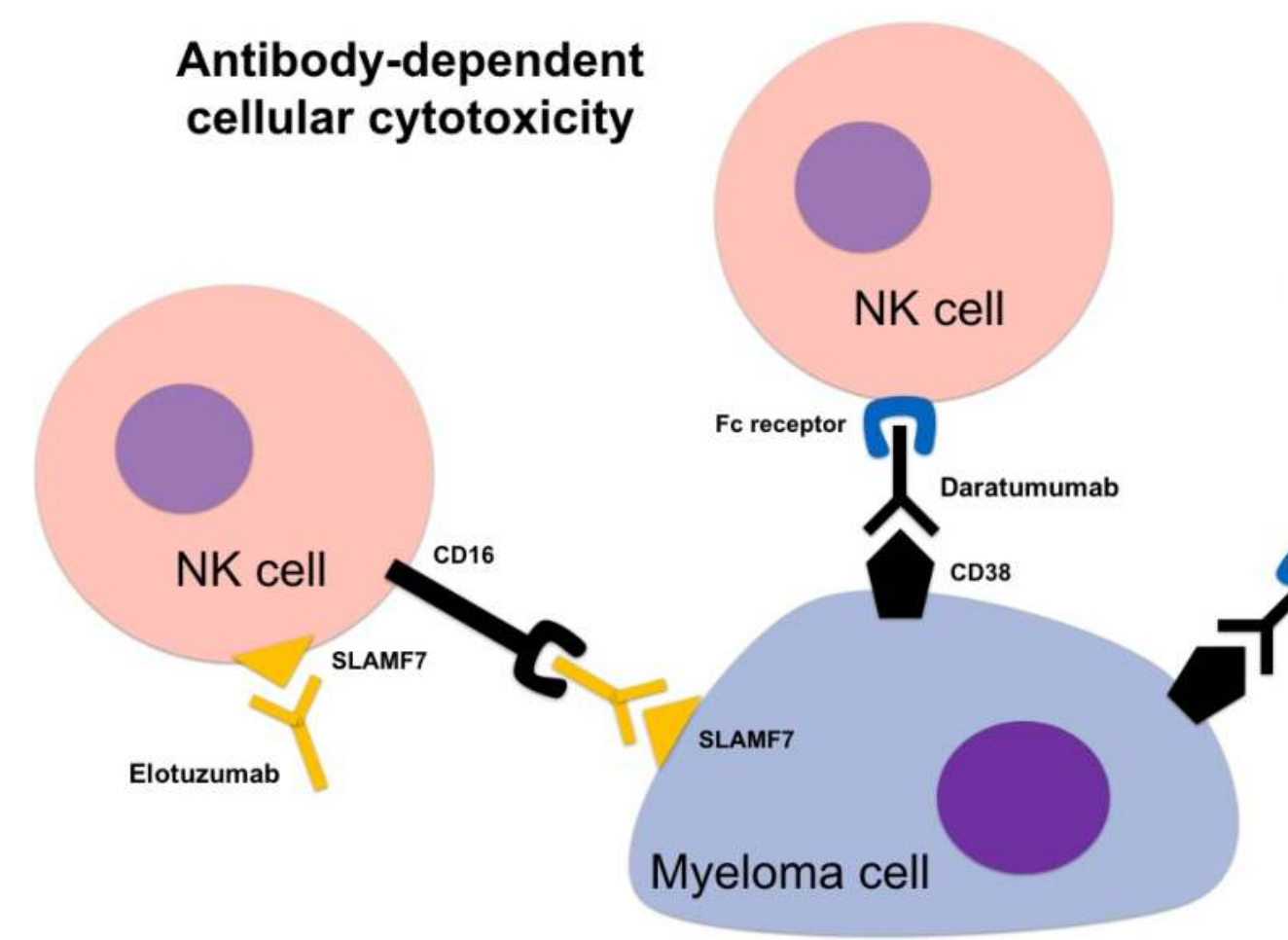

Complement-dependent

cytotoxicity tibody-dependent cellular phagocytosis

REFERENCES

Janssen Biotech, Inc.

Bringhen S, De Wit, E \& Dimopoulos, MA (2017) New Agents in Wultiple Myeloma. An Examination of Safety Profiles. Clin Lymphom Leuk, 17, 391-407.e395.

Casneuf, T. et. al. (2017) Effects of daratumumab on natural killer cells Cof
myeloma. Blood Adv $1,2105-2114$. Costello, C. (2017) An update on the role of daratumumab in the Dimopoulos, M.A. et. al. (2016) Daratumumab, Lenalidomide, and D. M. (2018) ESCMID Study Group for Infections in Compromised Hosts (ESGICH) Consensus Document on the safety of targeted and biological or myeloid cells surface antigens $[$ IIII: $\mathrm{CD} 222, \mathrm{CD} 30, \mathrm{CD} 33, \mathrm{CD} 38, \mathrm{CD} 40$, SLAMF-7 and CCR4). Clin Microbiol Infect, 24 Suppl 2, S83-S94.

Lonial, S. et. al. (2016) Daratumumab monotherapy in patients with treatment-refractory multiple myeloma (SIRIUS): an open-label,

Palumbo, A. et. al. (2016) Daratumumab, Bortezomib, and Plesner, T. et. al. (2016) Phase $1 / 2$ study of daratumumab, lenalidomide, . Tzogani, K. et. al. (2018) EMA Review of Daratumumab for the
Treatment of Adult Patients with Multiple Myeloma. Oncologist, 23, 594-

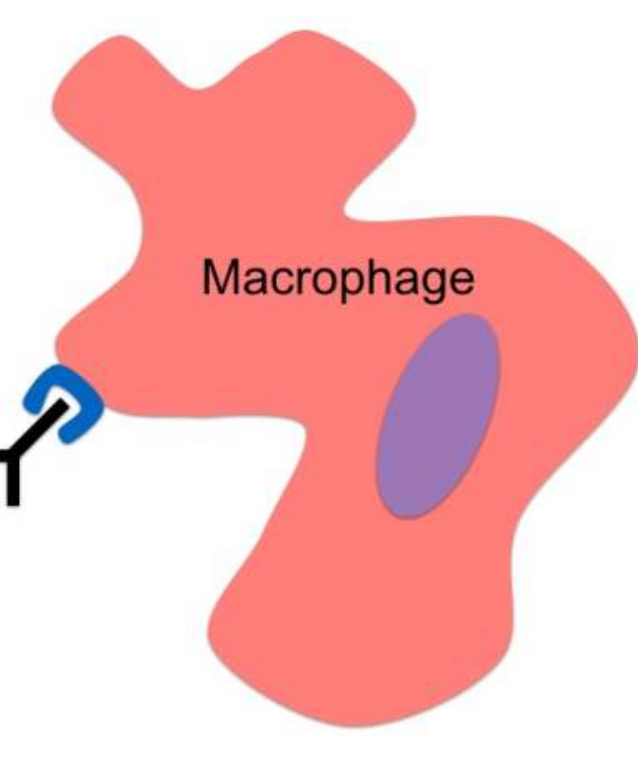

